\title{
Ethanol extracts of black pepper or turmeric down-regulated SIRT1 protein expression in Daudi culture cells
}

\author{
YURI NISHIMURA* ${ }^{*}$ YASUKO KITAGISHI, HITOMI YOSHIDA, NAOKO OKUMURA and SATORU MATSUDA* \\ Department of Environmental Health Science, Nara Women's University, Nara 630-8506, Japan
}

Received April 1, 2011; Accepted May 11, 2011

DOI: $10.3892 / \mathrm{mmr} .2011 .487$

\begin{abstract}
SIRT1 is a mammalian candidate molecule involved in longevity and diverse metabolic processes. The present study aimed to determine the effects of certain herbs and spices on SIRT1 expression. Human cell lines Daudi, Jurkat, U937 and K562 were cultured in RPMI-1640. Herb and spice powders were prepared and the supernatants were collected. RT-PCR was used to quantify the expression level of the gene. Protein samples were then analyzed by Western blotting. Western blotting revealed the down-regulation of SIRT1 protein expression in Daudi cells treated with extracts of black pepper or turmeric. On the other hand, the effect on the SIRT1 gene expression examined by reverse transcription polymerase chain reaction was unaltered. In conclusion, component(s) of certain herbs and spices may induce the down-regulation of SIRT1 protein.
\end{abstract}

\section{Introduction}

Mammalian SIRT1 (the closest relative of yeast Sir2) is an $\mathrm{NAD}^{+}$-dependent deacetylase that plays a role in longevity and diverse metabolic processes (1). The enzymatic activity may be regulated by cellular energy, and SIRT1 may serve as a key regulator of certain obesity comorbidities related to antioxidant status (2). Transgenic mice with an overexpression of SIRT1 exhibit normal insulin sensitivity but decreased food intake and locomotor activity, resulting in decreased energy expenditure (3). Furthermore, SIRT1 transgenics exhibit improved glucose tolerance due to decreased hepatic glucose production and increased adiponectin levels without changes in body weight or composition. SIRT1 primes the organism for metabolic adaptation to insulin resistance and decreases whole-body energy requirements. SIRT1 overexpression also

Correspondence to: Dr Satoru Matsuda, Department of Environmental Health Science, Nara Women's University, Kita-Uoya Nishimachi, Nara 630-8506, Japan

E-mail:smatsuda@cc.nara-wu.ac.jp

*Contributed equally

Key words: SIRT1, longevity, turmeric, black pepper, down-regulation reduces the level of oxygen consumption, which is associated with non-alcoholic fatty liver disease (NAFLD). Conversely, the expression of SIRT1 protein is significantly decreased in NAFLD induced by a high-fat diet in rats (4). A hypocaloric diet up-regulates SIRT1 expression, since it is inversely associated with total antioxidant capacity and directly related to nitric oxide and mitochondrial oxidation. Alterations in food intake, such as caloric restriction, modulate the expression of SIRT1 protein (5), which may provide novel targets for treating certain diseases associated with oxidative stress such as obesity. Accordingly, SIRT1 may be a useful therapeutic target for age-related diseases, including metabolic disorders.

Certain herbs have been suggested to possess a wide variety of beneficial activities. Herbal medications are currently widespread for clinical use in therapy, as the herbs have relatively mild bioavailability and low toxicity (6). However, insufficient scientific data are available on the efficacy of herbal therapies. The precise mechanisms for the effects of these herbs remain to be elucidated, and limited data and little convincing evidence have been provided at the molecular level (7). Therefore, basic research and development with the objective of elucidating the mechanisms of action underlying the herbal effects should be a priority. Resveratrol, a well-known SIRT1 activator, is considered to be one of the beneficial components contained in red wine (8). In addition, the methanol extract of Myristica fragrans Houtt (nutmeg) has been reported to induce the down-regulation of SIRT1 mRNA expression in Jurkat cells (9). We hypothesized that certain herbs or spices affect the expression of SIRT1, since it is well known that certain herbs are administered as an alternative treatment for preventing metabolic diseases. Therefore, we tested the in vitro effect of various herbs on the expression of SIRT1 in cultured cells.

\section{Materials and methods}

Cell culture. The human cell lines Daudi, Jurkat, U937 and K562 were maintained in RPMI-1640 supplemented with $10 \%$ fetal bovine serum (FBS), penicillin and streptomycin at $37^{\circ} \mathrm{C}$ in a humidified atmosphere containing $5 \% \mathrm{CO}_{2}$.

Extract preparation. Herb and spice powders were purchased at a food market in Japan. The powders were dissolved in $80 \%$ ethanol and subsequently diluted in $40 \%$ ethanol at a stock concentration of $50 \mathrm{mg} / \mathrm{ml}$. The mixtures were vortexed 


\section{$\begin{array}{llllllll}1 & 2 & 3 & 4 & 5 & 6 & 7 & 8\end{array}$}

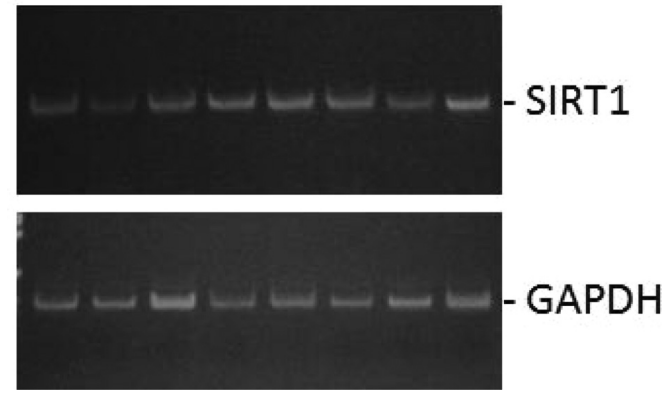

Figure 1. Semiquantitative RT-PCR was performed using primers specific to SIRT1 or GAPDH on 100 ng total RNA prepared from Jurkat cells treated with (lanes 2-8: garlic, red pepper, cinnamon, coriander, turmeric, basil and black pepper, respectively) or without (lane 1) herb extracts at a final concentration of $50 \mu \mathrm{g} / \mathrm{ml}$ for $48 \mathrm{~h}$. The specific expression was determined in relation to the expression of the housekeeping gene GAPDH, which was used as an internal loading control. At least four independent experiments were carried out and typical paired results are documented.

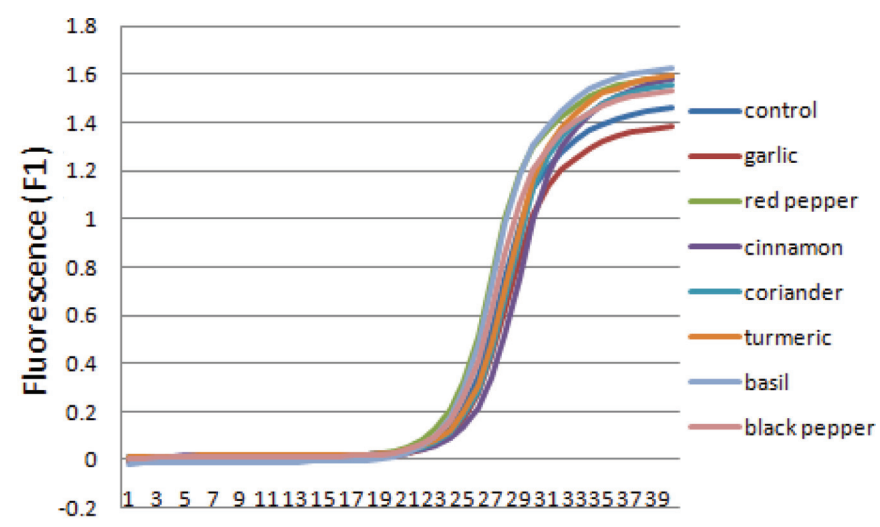

Cycle number

Figure 2. Fluorescence data for PCR amplification plots of SIRT1 in Daudi cells stimulated by each herb or spice. Data were generated by the ThermalCycler software on Illumina eco real-time PCR detection system. No product was amplified in the no-template sample or when reverse transcriptions were omitted. Similar results were obtained when PCR products of U937, Jurkat and K562 cells were analyzed.

rigorously for $3 \mathrm{~min}$ followed by $3 \mathrm{~min}$ sonication. Following centrifugation $(1500 \mathrm{x} \mathrm{g}, 5 \mathrm{~min})$, the supernatants were collected and stored at $-20^{\circ} \mathrm{C}$ until use. For the cell treatments, 0.5-10.0 $\mu \mathrm{l}$ were added to $1 \mathrm{ml}$ of cell culture medium.

Reverse transcriptase polymerase chain reaction. SIRT1 and GAPDH messenger ribonucleic acids (mRNAs) were analyzed by semiquantitative Reverse transcriptase polymerase chain reaction (RT-PCR). Total ribonucleic acid (RNA) was extracted by an RNA isolation kit (TAKARA BIO, Takara, Japan). Total RNA (2 $\mu \mathrm{g})$ was reverse-transcribed using a Phusion RT-PCR kit (New England BioLabs, Finnzymes, Finland) according to the manufacturer's protocol. Cyclebased PCR was used to semiquantitate the SIRT1 gene level. Semiquantitative RT-PCR was performed using primers specific to SIRT1 or GAPDH on $100 \mathrm{ng}$ total RNA prepared from Jurkat cells treated with garlic, red pepper, cinnamon, coriander, turmeric, basil and black pepper, respectively,

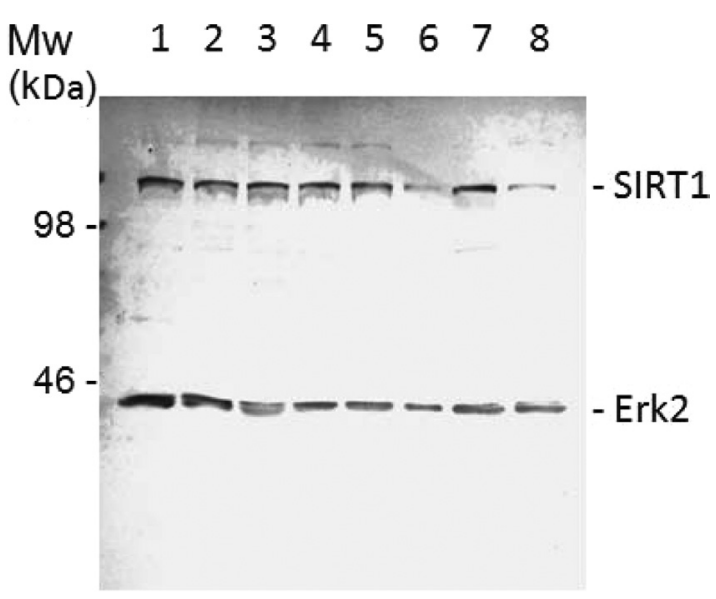

Figure 3. Black pepper or turmeric extracts reduced the expression of SIRT1 protein. Daudi cells were treated with (lanes 2-6: garlic, red pepper, cinnamon, coriander, turmeric, basil and black pepper, respectively) or without (lane 1) extracts of herbs at a final concentration of $50 \mu \mathrm{g} / \mathrm{ml}$ for $72 \mathrm{~h}$. Following treatment, cell lysates were isolated and the levels of SIRT1 protein were detected by Western blot analysis using an anti-SIRT1 antibody. Western blotting with anti-Erk2 antibody revealed equal levels of protein loading. At least three independent experiments were carried out, and typical paired results are shown.

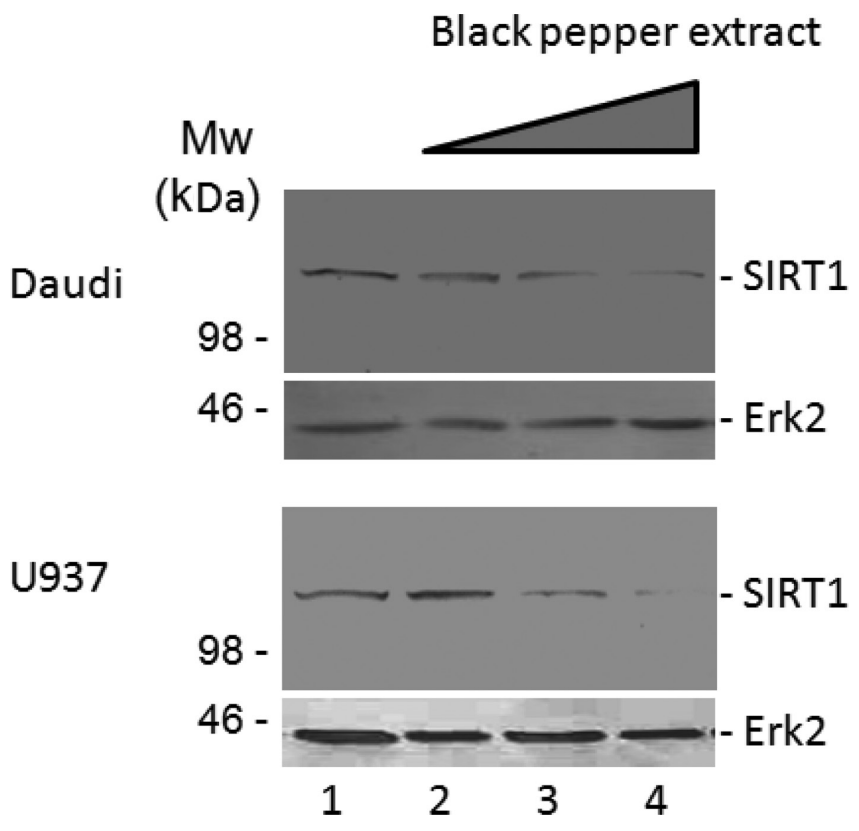

Figure 4. Dose-dependent inhibition of SIRT1 protein expression by black pepper. Daudi cells (upper panel) or U937 cells (lower panel) were treated with (lane 2) or without (lane 1) black pepper extract at final concentrations of $25 \mu \mathrm{g} / \mathrm{ml}, 50 \mu \mathrm{g} / \mathrm{ml}$ (lane 3) and $100 \mu \mathrm{g} / \mathrm{ml}$ (lane 4) for $72 \mathrm{~h}$. Protein levels were detected by Western blot analysis using an anti-SIRT1 antibody as in Fig. 3. Western blotting with anti-Erk2 antibody also revealed equal levels of protein loading in each experiment.

or without herb extracts at a final concentration of $50 \mu \mathrm{g} / \mathrm{ml}$ for $48 \mathrm{~h}$. GADPH was also used as an internal loading control. The samples were determined within 3 months of collection. The primers used for the PCR were: SIRT1 Fw: AAGGTTCATTTGTATGATA, SIRT1 Rv: AATTCTTAGCACCAAGTAT (expected size: 380 bp); GAPDH Fw: TCCCATCACCATCTTCCA, GAPDH Rv: 
CATCACGCCACAGTTTCC (expected size: 376 bp). For real-time PCR, the reactions were performed in a real-time PCR system (Illumina, San Diego, CA, USA) using KAPA SYBR FAST Reaction Mix (Genetics, Japan). Thermo-cycling was performed according to the manufacturer's instructions at $60^{\circ} \mathrm{C}$ annealing temperature in a final volume of $10 \mu \mathrm{l}$ including Taq DNA polymerase. To correct for differences in both RNA quality and quantity between samples, data were normalized using the ratio of the target cDNA concentration to that of GAPDH.

Western blot analysis. Equal amounts of protein samples were used for Western blot analysis using anti-SIRT1 (Abnova) and anti-Erk2 (Epitomics) antibodies, and quantified by densitometry. All of the Western blots were repeated at least three times and the representative data were shown.

\section{Results and Discussion}

Extracts of garlic, red pepper, cinnamon, coriander, turmeric, basil and black pepper were added into cell culture media of Daudi, Jurkat, U937 and K562 cells and the levels of genes, including SIRT1, were examined. RT-PCR analysis was employed to quantify the expression level of the gene. Total RNA was isolated $48 \mathrm{~h}$ after herbal extract treatment for the detection of SIRT1 and the levels of mRNA were determined by conventional semiquantitative RT-PCR. As shown in Fig. 1, the SIRT1 gene expression level was not altered by the administration of herb or spice extracts at final concentrations of $50 \mu \mathrm{g} / \mathrm{ml}$, compared with the untreated ethanol vehicle. The expression of SIRT2 (data not shown) and the housekeeping gene GAPDH were also unaltered (Fig. 1). Similar results were obtained from the quantitative real-time PCR analysis (Fig. 2). Differences between the gene expression profiles of Daudi, Jurkat, U937 and K562 cells were insignificant (data not shown). To exclude the possibility of carry-over DNA contamination, reactions containing the RT-PCR reagents, including primers without sample RNA, were preformed as negative controls. No such RNA contamination was detected (data not shown).

To examine the expression status at the protein level, Western blot analysis was used to analyze the SIRT1 protein in the cells stimulated by herbs and spices. As shown in Fig. 3, the black pepper and turmeric extracts markedly reduced the protein expression of SIRT1, but not Erk2 protein, in Daudi cells, when cell cultures had been maintained in the spice stimulation for $72 \mathrm{~h}$. The SIRT1 protein expression down-regulated by a number of the herb extracts approximately agreed with the result of early-harvested ( $48 \mathrm{~h}$ after herbal stimulation) cells (data not shown). After treating the cells with a set of concentrations of black pepper, Western blotting revealed that SIRT1 protein, but not Erk2, expression was decreased with the increasing concentrations of the extracts. Final concentrations of $100 \mu \mathrm{g} / \mathrm{ml}$ of turmeric extract inhibited SIRT1 expression by more than $80 \%$ in the Daudi and U937 cells (Fig. 4). We then addressed the question of whether capsaicin, which is a component of pepper, is capable of reducing SIRT1 expression. However, capsaicin did not down-regulate SIRT1 (data not shown).

An increased expression of SIRT1 is capable of extending the life span of model organisms and it appears that the activity of Sir2 (a homolog of SIRT1) is required for the lifeextending benefits of caloric restriction in organisms such as yeast and flies (10). SIRT1 levels are regulated by microRNAs (miRNAs) that have emerged as significant metabolic regulators (1). SIRT1 deacetylase may be involved in some manner in protecting cells from nutrient stress. It has been noted that chronic caloric restriction augments the level of SIRT1 (11). Conversely, SIRT1 levels are decreased in chronic inflammatory conditions and aging where oxidative stress occurs. Oxidants covalently modify SIRT1, decreasing enzymatic activity and demarcating the protein for proteasomal degradation, which has implications in inflammatory conditions (12). Recently, it has been reported that ionizing radiation triggers the down-regulation of SIRT1 protein expression but not the transcript level, indicative of post-transcriptional cleavage of the protein (13). Investigators have proposed that SIRT1 down-regulation was directly or indirectly associated with p38 kinase as opposed to the NF-kB pathway. Since the proteasome is responsible for the removal of oxidatively damaged proteins in the cytosol and nucleus during oxidative stress and aging (14), it is plausible that SIRT1 protein is degraded by the proteasome pathway, in which case herbs and spices such as black pepper or turmeric may accelerate the degradation. Our results reveal that component(s) of black pepper or turmeric induced down-regulation of the SIRT1 protein, suggesting that including a large amount of these spices in one's daily diet may be harmful to health. Further studies, including in vivo experiments, are required to elucidate the precise molecular mechanisms of these herbs and spices.

\section{Acknowledgements}

This study was supported by grants-in-aid from the Ministry of Education, Culture, Sports, Science and Technology in Japan and Nara Women's University Intramural Grant for Project Research.

\section{References}

1. Lee $\mathrm{J}$ and Kemper JK: Controlling SIRT1 expression by microRNAs in health and metabolic disease. Aging (Albany NY) 2: 527-534, 2010.

2. Crujeiras AB, Parra D, Goyenechea E and Martínez JA: Sirtuin gene expression in human mononuclear cells is modulated by caloric restriction. Eur J Clin Invest 38: 672-678, 2008.

3. Banks AS, Kon N, Knight C, et al: SirT1 gain of function increases energy efficiency and prevents diabetes in mice. Cell Metab 8: 333-341, 2008.

4. Deng XQ, Chen LL and Li NX: The expression of SIRT1 in non-alcoholic fatty liver disease induced by high-fat diet in rats. Liver Int 27: 708-715, 2007.

5. Amaral ME, Ueno M, Oliveira CA, et al: Reduced expression of SIRT1 is associated with diminished glucose-induced insulin secretion in islets from calorie-restricted rats. J Nutr Biochem: Aug 27, 2010 (Epub ahead of print).

6. Abad MJ, Bedoya LM and Bermejo P: An update on drug interactions with the herbal medicine Ginkgo biloba. Curr Drug Metab 11: 171-181, 2010.

7. Okumura N, Nishimura Y, Yoshida H, Nagata Y, Kitagishi Y and Matsuda S: Ethanol extract of herb Sage suppressed the TIMP-1 expression in lymphoid culture cells. Asia J Sci Technol 3: 64-66, 2010.

8. Tsukamoto T, Nakata R, Tamura E, et al: Vaticanol C, a resveratrol tetramer, activates PPARalpha and PPARbeta/delta in vitro and in vivo. Nutr Metab (Lond) 7: 46, 2010. 
9. Chirathaworn C, Kongcharoensuntorn W, Dechdoungchan T, Lowanitchapat A, Sa-nguanmoo $\mathrm{P}$ and Poovorawan $\mathrm{Y}$ Myristica fragrans Houtt. methanolic extract induces apoptosis in a human leukemia cell line through SIRT1 mRNA downregulation. J Med Assoc Thai 90: 2422-2428, 2007.

10. Allard JS, Perez E, Zou S and De Cabo R: Dietary activators of Sirt1. Mol Cell Endocrinol 299: 58-63, 2008.

11. Yu W, Fu YC, Chen CJ, Wang X and Wang W: SIRT1: a novel target to prevent atherosclerosis. J Cell Biochem 108: 10-13, 2009.
12. Caito S, Rajendrasozhan S, Cook S, et al: SIRT1 is a redoxsensitive deacetylase that is post-translationally modified by oxidants and carbonyl stress. FASEB J 24: 3145-3159, 2010.

13. Hong EH, Lee SJ, Kim JS, et al: Ionizing radiation induces cellular senescence of articular chondrocytes via negative regulation of SIRT1 by p38 kinase. J Biol Chem 285: 1283-1295, 2010

14. Breusing $\mathrm{N}$ and Grune T: Regulation of proteasome-mediated protein degradation during oxidative stress and aging. Biol Chem 389: 203-209, 2008. 